\title{
The ALPPS Approach: Should We Sacrifice Basic Therapeutic Rules in the Name of Innovation?
}

\author{
J. Figueras $\cdot$ J. Belghiti
}

Published online: 23 April 2014

(c) Société Internationale de Chirurgie 2014

Tolerance to extended liver resection has been dramatically improved with the development of preoperative liver volume modulation techniques using portal vein occlusion. The ALPPS approach (Associating Liver Partition with Portal Vein Ligation for Staged Hepatectomy), which associates in situ splitting to portal vein ligation, theoretically provides two main advantages compared to traditional liver volume modulation strategies. First, both enhanced portal flow deprivation in the future resected liver and accentuated inflammatory response are thought to induce faster regeneration compared to traditional strategies, and thus reduce dropout in patients with marginally resectable disease. Second, adding parenchymal transection would allow triggering liver regeneration in the future liver remnant when other classical strategies are likely to be ineffective. Hence, this strategy would appear relevant for patients with portal vein thrombosis, as recently suggested [1]. It has been almost two years since the pioneering publication of Schnitzbauer et al. [2] describing their breakthrough surgical strategy, and many centers around the world have since reported their own experience. Some reports have aimed at discussing and refining both techniques and indications, whereas others have focused on alerting readers to possible issues brought about by this audacious approach. Although much ink has flowed, most concerns have remained only speculative, and we still await midterm and long-term results.

J. Figueras $(\bowtie)$

Department of Surgery, Dr Josep Trueta Hospital, Girona, Spain e-mail: info@jfigueras.net

\section{J. Belghiti}

Department of HPB Surgery \& Liver Transplantation, Beaujon Hospital, University Paris, Paris, France
In the present issue of World Journal of Surgery, we have the privilege of comparing two major complementary papers on the ALPPS approach. The first study arises from the group of Clavien [3] and compares classical two-stage liver resections and ALPPS approach using rigorous retrospective methodology. In this setting, the authors provide solid evidence that ALPPS is associated with higher liver hypertrophy, allows higher resectability rates, and thus offers a better chance of complete resection in patients with primarily unresectable liver tumors. Although these results represent a clear answer to an on-going debate regarding the theoretical superiority of this approach, the observed low rates of postoperative liver insufficiency do not compensate for other technical complications that frequently occur with this strategy. In that sense, the reported prohibitive morbidity and mortality rates certainly call into question the clinical applicability of the procedure, especially in low volume liver units without previous practice of two-step liver surgery or with only limited experience in living donor liver transplantation.

This is potentially a particularly serious issue in consideration of the debatable oncologic advantages of the ALPPS approach. Indeed, the second study, from Oldhafer et al. [4], which arises from Germany, focuses on the longterm outcomes of this approach in patients with unresectable colorectal liver metastases. In their brief but honest series report, the authors indicate that no postoperative mortality occurred and that $\mathrm{R} 0$ resection was achieved in all cases. Nevertheless, as many as $86 \%$ of the patients experienced recurrence, with at least one extrahepatic location in every case. Whether the extreme hypertrophy itself promotes recurrence remains uncertain and will require further investigation.

If one considers that ALPPS should be used in order to go even further than classical two-step strategies, the 
observed results clearly call into question the benefits of achieving complete resection in the subset of patients with a poor oncologic prognosis; in addition, the findings emphasize that anticipating the natural history of the disease should now be the primary focus of liver surgeons [5].

\section{References}

1. Vennarecci G, Laurenzi A, Santoro R et al (2013) The ALPPS procedure: a surgical option for hepatocellular carcinoma with major vascular invasion. World J Surg. doi:10.1007/s13304-0130214-3
2. Schnitzbauer AA, Lang SA, Goessmann H et al (2012) Right portal vein ligation combined with in situ splitting induces rapid left lateral liver lobe hypertrophy enabling 2-staged extended right hepatic resection in small-for-size settings. Ann Surg 255:405-414

3. Schadde E, Ardiles V, Slankamenac K et al (2014) ALPPS offers a better chance of complete resection in patients with primarily unresectable liver tumors. Results of a multicenter analysis. World J Surg. doi:10.1016/j.ijscr.2013.07.030

4. Oldhafer KJ, Donati M, Jenner RM et al (2014) ALPPS for patients with colorectal liver metastases: effective liver hypertrophy, but early tumor recurrence. World J Surg. doi:10.1007/ s11888-013-0159-4

5. Cauchy F, Aussilhou B, Dokmak S et al (2012) Reappraisal of the risks and benefits of major liver resection in patients with initially unresectable colorectal liver metastases. Ann Surg 256:746-752 